\title{
Build Reliable and Efficient Neuromorphic Design with Memristor Technology
}

\author{
Bing Li ${ }^{*}$ \\ ECE Dept., Duke University, Durham, NC,USA \\ Army Research Office, Research Triangle Park,USA \\ bing.li.ece@duke.edu \\ Chenchen Liu \\ ECE Dept., Clarkson Univesity \\ Potsdam, NY, USA \\ chliu@clarkson.edu
}

\author{
Bonan Yan \\ ECE Dept., Duke University, Durham, NC, USA \\ bonan.yan@duke.edu
}

\author{
Hai (Helen) Li \\ ECE Dept., Duke University \\ Durham, NC, USA \\ hai.li@duke.edu
}

\begin{abstract}
Neuromorphic computing is a revolutionary approach of computation, which attempts to mimic the human brain's mechanism for extremely high implementation efficiency and intelligence. Latest research studies showed that the memristor technology has a great potential for realizing power- and area-efficient neuromorphic computing systems (NCS). On the other hand, the memristor device processing is still under development. Unreliable devices can severely degrade system performance, which arises as one of the major challenges in developing memristor-based NCS. In this paper, we first review the impacts of the limited reliability of memristor devices and summarize the recent research progress in building reliable and efficient memristor-based NCS. In the end, we discuss the main difficulties and the trend in memristor-based NCS development.
\end{abstract}

\section{ACM Reference Format:}

Bing Li, Bonan Yan, Chenchen Liu, and Hai (Helen) Li. 2019. Build Reliable and Efficient Neuromorphic Design with Memristor Technology. In ASPDAC '19: 24th Asia and South Pacific Design Automation Conference (ASPDAC '19), January 21-24, 2019, Tokyo, Japan. ACM, New York, NY, USA, 6 pages. https://doi.org/10.1145/3287624.3288744

\section{INTRODUCTION}

In recent years, the data-centric cognitive computations have been widely applied in various applications at different scales, such as scientific computing, cloud computing, and consumer electronics. Conventional von Neumann architecture can only provide the limited data processing capability and poor scalability for the datacentric applications. Thus, extensive research studies have been conducted on developing neuromorphic computing system (NCS)

\footnotetext{
${ }^{*}$ Author is supported by the NRC Associate Fellowship Award
}

Permission to make digital or hard copies of all or part of this work for personal or classroom use is granted without fee provided that copies are not made or distributed for profit or commercial advantage and that copies bear this notice and the full citation on the first page. Copyrights for components of this work owned by others than ACM must be honored. Abstracting with credit is permitted. To copy otherwise, or republish to post on servers or to redistribute to lists, requires prior specific permission and/or a fee. Request permissions from permissions@acm.org.

ASPDAC '19, January 21-24, 2019, Tokyo, Japan

(C) 2019 Association for Computing Machinery.

ACM ISBN 978-1-4503-6007-4/19/01 . \$15.00

https://doi.org/10.1145/3287624.3288744 which mimics the biological neural system and is viewed as the next-generation computing paradigm.

The emerging memristor technology has been regarded as one of the most promising techniques to implement the energy-efficient and low-cost NCS [1-3]. Memristor devices feature the excellent merits including the nanoscale dimension, the nonvolatile storage, the high capacity of storing multiple logic bits per cell and the low access energy $[4,5]$. In addition, the memristor crossbar structure can naturally realize the vector-matrix multiplicationthe elementary operation in data-centric algorithms. As illustrated in Figure 1(a), in a memristor crossbar, the wordlines (WLs) and bitlines (BLs) intersect with each other, forming the rows and columns of a matrix. The resistance state of a memristor (referred as memristance) corresponds to an element of the matrix.

Massive studies have been carried out in developing the memristorbased NCS. For example, Liu et al. [6] presented an implementation for feed-forward neural networks. Hu et al. [2] investigated the mapping strategy for the dot-product engine by considering the limited bit precision of memristor. Song et al. [3] explored various data flow designs to enhance the computation efficiency. The primary objective of these prior works was to improve the overall energy efficiency and operation speed. The reliability issue, as one of the major challenge on the way of broad applications of the memristor-based NCS, hasn't been granted the sufficient attention.

In addition to the common flaws in memory processing such as stuck-on/-off defects and process variations, the memristor-based NCS could also suffer from severe performance degradation due to the defects in devices and/or the variations in training [7-10]. For example, Liu et al. [8] previously showed that as the device defect rate increases to $20 \%$, the classification accuracy of a two-layer neural network for the MNIST database drops quickly from $92.6 \%$ to $39.4 \%$. A straightforward attempt was to adopt the existing faulttolerate techniques in memory, such as error correction code (ECC)

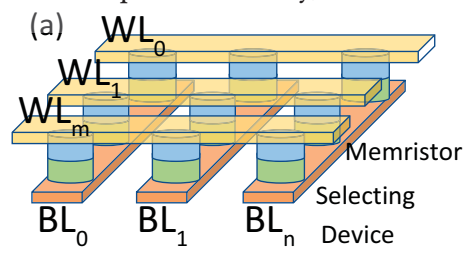

(b)

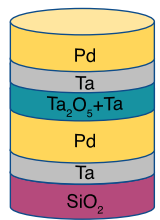

Figure 1: (a) An illustration of a memristor crossbar array; (b) the device stack of $\mathrm{TaO}_{\mathrm{x}}$-based memristor. 


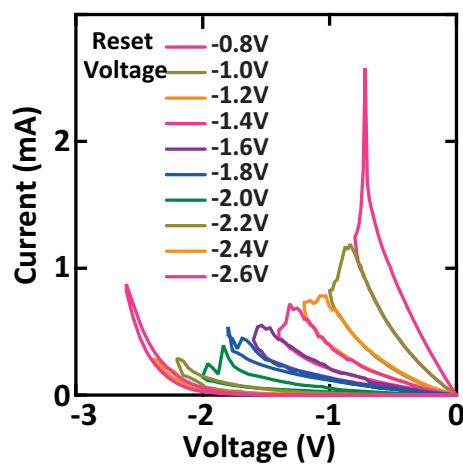

Figure 2: The measured $\mathrm{I}-\mathrm{V}$ curve of $\mathrm{TaO}_{\mathrm{x}}$ device [10].

and redundancy, to enhance the reliability of the memristor-based NCS [11-14]. The exploration revealed that these methods are not sufficient enough to recover the degraded computation accuracy of the neural network applications. Moreover, the induced computation overhead and additional circuit components might cause new problems. How to build reliable and efficient neuromorphic design with memristor technology emerges as a crucial problem to solve.

\section{BACKGROUND}

\subsection{Memristor}

As early as in 1971, Professor Leon Chua predicted the existence of memristor, the fourth fundamental circuit element in addition to resistor, capacitor and inductor [15]. The resistance value of a memristor, aka, memristance $M$, reflects the change of magnetic flux resulted by the flow of electrical charge, i.e., $M=\mathrm{d} \phi / \mathrm{d} q$ [15]. However, it took researchers decades to experimentally approve the existence of memristor. In 2008, Hewlett Packard Enterprise (HPE) Labs successfully demonstrated memristive features with a nanoscale device [16]. Since then, memristor devices have been widely investigated. Benefiting from it simple structure, excellent scalability, two-direction programming and fast read/write speed, the applications of memristor have been extended from storage, main memory to on-chip caches [4, 17, 18]. For instance, Intel CrossPoint employs a 2-layer memristor for the server storage applications [5].

Memristor array structure is also commonly referred as resistive random access memory (ReRAM or RRAM). Memristor device usually consists of a metal-oxide material layer sandwiched between two metal electrode layers. Various metal-oxide materials, e.g. TiOx [19], WOx [9] and HfOx [20] have been utilized to realize the memristors devices. Here, we illustrate the basic device structure of a $\mathrm{TaO}_{\mathrm{x}}$-based device in Figure 1(b). By properly applying a voltage excitation at the two electrical terminals across a memristor, its memristance can continuously switch between the lowest value $\left(R_{\mathrm{LRS}}\right)$ and the highest memristance $\left(R_{\mathrm{HRS}}\right)$.

There are two operations in memristor to change its states by applying the voltages with different directions: "On" denotes to program a device from $R_{\mathrm{HRS}}$ to $R_{\mathrm{LRS}}$, which is also called as SET; "Off" is the opposite operation that switches a device to $R_{\text {HRS }}$. The procedure is also called as RESET. Memristor devices often possess non-linear current-voltage $(I-V)$ characteristics [21]. Figure 2

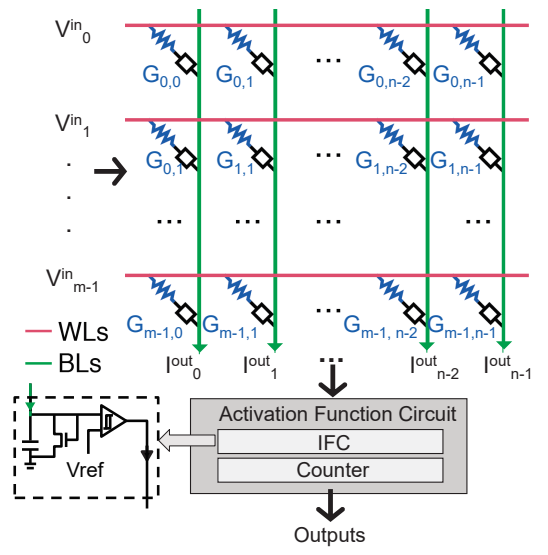

Figure 3: A spiking neuromorphic computing system that leverages memristor crossbar.

illustrates the measured $I-V$ curves of $\mathrm{TaO}_{\mathrm{x}}$-based device during RESET operations.

\subsection{Memristor-based NCS}

Figure 3 illustrates a memristor-based spiking NCS, in which the matrix-vector multiplication (MVM) is realized through memristor crossbar array. Assume $\mathrm{G}$ is the conductance values for an $m \times n$ memristor crossbar. According to Kirchoff's law, with an input vector represented by a set of input voltages $\vec{V}$, the output currents $\vec{I}$ of the crossbar are $\vec{I}=\mathrm{G} \vec{V}$, which are indeed the computation results. In this design, the output currents are then fed into the activation function circuit to generate the output spikes. There are two important components in the activation function circuit: the integrate \& fire circuit (IFC) that produces output spikes chain and the digital counter that calculates the output spike strength for subsequent circuits [6].

Previous research study [22] revealed that the MVM operation accounts for more than $70 \%$ computation resources in neural network execution. There have been many studies on memristor-based NCS, from the stand-alone MVM engine to the NN accelerator [2, 3, 19]. The progress demonstrates the potential of the memristor-based NCS in realizing low-cost, energy-efficient, and high-speed DNN computation.

Similar to many systems designed for neural network acceleration, the memristor-based NCS has two types of basic computation processes: training and recall. The training process adaptively tunes the matrix (more explicitly, the resistance values) to achieve acceptable accuracy for the tasks. As illustrated in Figure 4, the training process can be carried out through an open-loop (a) or a close-loop

(a)

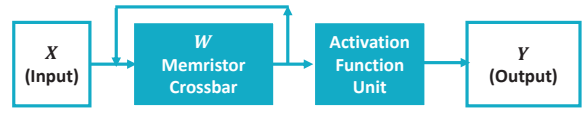

(b)

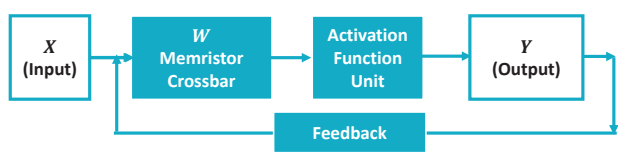

Figure 4: (a) The open-loop training and (b) the closed-loop training schemes [10]. 


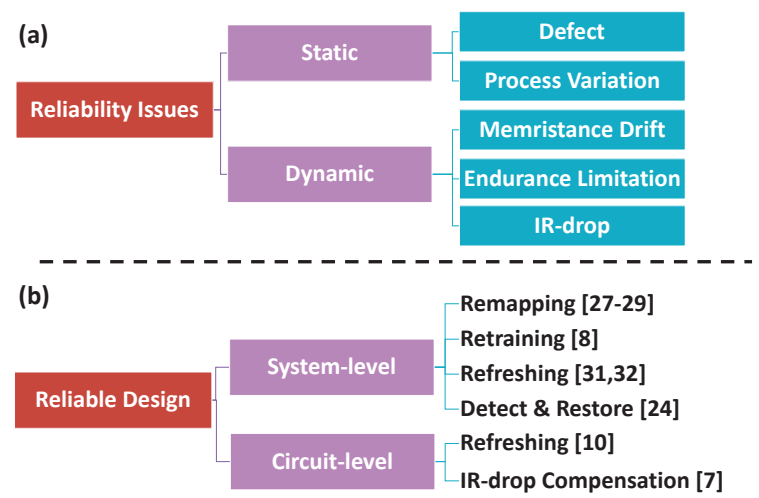

Figure 5: The categories of (a) the reliability issues in memristor technology and (b) the designs for enhancing reliability reviewed in this work.

(b) scheme. The open-loop training directly calculates the desired memristance values based on the stored weight patterns and iteratively calibrates the memristors. The close-loop scheme recursively updates the weights based on the feedback from the output $[7,19]$, which is more like the back-propagation in neural network training. The operation of the recall process is more straightforward by following the memristor operation mechanism described above.

\section{BUILD RELIABLE MEMRISTOR-BASED NCS}

Although memristor technology has demonstrated a great potential in developing the compact and energy-efficient embodiment for neuromorphic computing systems, various reliability issues induced by intrinsic device characteristics as well as immature fabrication process lead to the computation accuracy degradation and even the system failure. This has emerged as a major challenge in memristorbased NCS development.

In early stage, the memristor-based designs were primarily developed for memory applications. Traditional reliability enhancement techniques, such as error correction code (ECC) $[12,14]$ were deployed to detect errors and recover the corrupted data. The memristor-based NCS, on the contrary, demonstrated a completely different vulnerability on each individual devices. Moreover, the impact is not reflected by the logic error of device(s) but the computing results (e.g., system accuracy), which made the evaluation and design even more challenging. The situation started to gain attention lately and some research studies explored techniques for reliable and efficient memristor-based NCS.

\subsection{Reliability Issues}

Here, we separate the reliability issues in memristor-based NCS into categories-static and dynamic. The static issues refer to those that are due to the fabrication flaws and circuit structures. These issues exist once the devices are made and/or the circuit structure is determined. The situation will not change dramatically over time. For example, the fabrication defect is the most common reliability issue. It is unavoidable while the immature manufacturing process can further aggravate the situation. Some devices could freeze at the high resistance (stuck-off) or low resistance (stuck-on) and cannot be programmed. Another major static reliability issue is

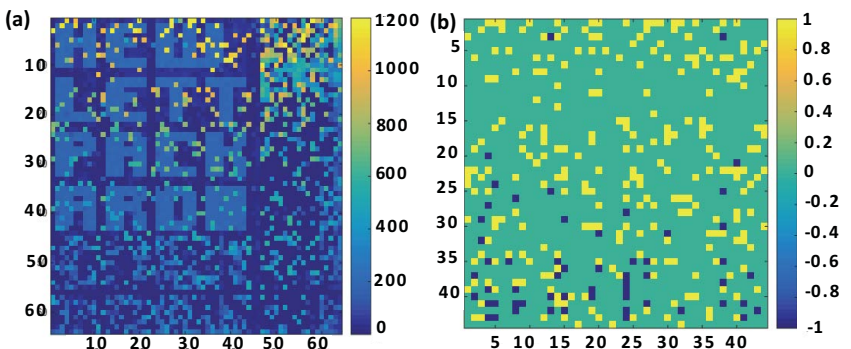

Figure 6: (a) The conductance distribution (in $\mu$ S) of a $64 \times 64$ memristor crossbar array, (b) the measured stuck on/off defects of a $44 \times 44$ matrix pattern [8].

process variation which in general is inevitable for the nano-scale technologies.

The dynamic reliability issues are usually involved with the nonideal behaviors of the memristor during operations [23-26]. The participated situations could be dynamically changed according to the operation conditions and might become even worse over time. The memristance drift is a typical scenario to represent the dynamic reliability issues: as normal recall operation always applies a voltage/current along a predetermined polarity across memristor devices, the actual memristances can gradually deviate from their targeted values. Such a change will be eventually reflected by the degradation of system performance. In addition, the limited endurance of the memristor leads to more and more defective devices, which further deteriorates the performance of the memristor-based NCS. At circuit level, the IR-drop along the interconnects in the memristor crossbar induces the bias of the output currents from the bit-lines from the desired values. The severity level is highly related to the array size and interconnect condition.

In addition to the investigation on physical material and device engineering, various system-level and circuit-level solutions were proposed to circumvent the static and dynamic reliability issues. We summarize the recent progress in building reliable memristor-based NCS designs, as shown in Figure 5.

\subsection{Overcome Static Reliability Issues}

In presence of the static reliability issues, the computing accuracy of the memristor-based NCS can be significantly deteriorated. Let's take the defect failure as the example. Figure 6(a) is the memristance distribution by measuring a $44 \times 44$ pattern in a $64 \times 64$ onetransistor-one-resistor (1T1R) array provided by HPE Labs [8]. The measurement shows a relative low yield of $84 \%$. Figure 6(b) shows that the measured distribution of stuck-on and stuck-off defects in the $44 \times 44$ region. Here, the yellow and dark-blue dots represent the stuck-on and stuck-off defects, respectively. Among all the defects, the stuck-on defects account for $18.4 \%$.

Due to the high defect ratio, the classification accuracy when implementing a neural network on a memristor-based NCS could be much lower than the ideal value obtained from its algorithm-level counterpart. For example, for a two-layer fully-connected neural network tested on MNIST dataset, the classification accuracy decreases from $92.6 \%$ to $59.7 \%$ when the defect rate increases to $10 \%$. Furthermore, after taking the endurance effect into the consideration, the intensive operations can gradually exhaust the lifetime of 
memristors. As a result, the defect rate grows up and thereby the accuracy degrades over time. Common approaches to address the static reliability issues include remapping and retraining.

3.2.1 Remapping. Huangfu et al. [27] used a couple of memristor crossbars to represent a weight matrix in the memristor-based NCS. They then proposed to map one weight element to the available devices in these crossbar arrays, in order to reduce the total number of errors of a weight matrix. The currents drawn from the corresponding BLs of these crossbar arrays are combined to approximate the computation results, and thereby alleviate the impact of defects on the system performance. As can be seen that the technique requires to modify output circuit to support the computation.

The remapping method proposed by Xia et al. [28, 29] utilized the sparsity of neural networks to guide the remapping design. It re-ordered the columns/rows of the weight matrix and mapped the zero elements to the devices with stuck-off defects. Compared to [27], this approach can dramatically reduce the circuit overhead.

3.2.2 Retraining. The remapping technique utilizes the same neural network configuration and adapts the weight mapping according to the defect maps. Liu et al. [8] took the retraining approach, which tends to obtain the network weight configuration optimized for each memristor-based NCS. The method was developed based on the observation that different weights affects the system performance in different extents. The weight significance is introduced to denote the importance of a weight element, which is quantified by its gradient value with respect to the global error. The weights in an array are then classified into significant and insignificant groups: Defects on the significant weights severely affect the system performance whereas the degradation of the accuracy is tiny when the insignificant weights fall at defective devices. In the retraining algorithm, the memristor-based NCS masks the defected device from the chip testing stage and only updates those the tunable weights. The retraining process is executed iteratively until the classification accuracy is recovered. If there are too many defects in the crossbar, a small portion of redundant columns can assist to store the significant weights.

Figure 7 compares the evaluated results before and after applying the retraining design for a two-layer neural network. According to the algorithm testing, the classification accuracy of this model on MNIST dataset is $92.64 \%$. The simulation result in Figure 7(a) shows that $20 \%$ defect rate can cause the accuracy drop to $42.5 \%$.
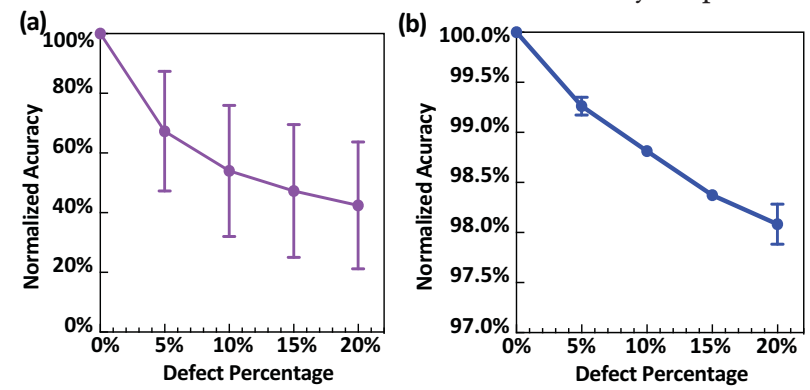

Figure 7: (a) The memristor device defects severely degrade the classification accuracy of a two-layer neural network; and (b) the recognition accuracy is recovered after applying the retraining technique [8].
The figure depicts the normalized accuracy ( $y$-axis) which denotes the accuracy ratio of the real device to the ideal memristor device, while the a-axis is the percentage of the defect in the memristor crossbar. Figure 7(b) shows that the retraining can successfully recover the classification accuracy to a reasonable level, for the same memristor-based NCS under the aggressive defect rate of $20 \%$.

\subsection{Mitigate Dynamic Reliability Issues}

As the dynamic reliability issues feature dynamical changes according to the operation conditions, how to mitigate the impact is very challenging. For example, the impact of the IR-drop indeed is related to not only the array design but all the operation condition such as the input pattern and weight distribution. It can severely degrade the computation accuracy and limit the scalability of memristor arrays. Moreover, the state drift per operation will accumulate through iterations and ultimately result in the corruption of the memristor state. Due the high complexity of the problem, various solutions have been proposed to address the dynamic reliability issues in memristor-based NCS.

3.3.1 IR-Drop compensation. The IR-drop along the interconnects of memristor array is a major source of the inconsistency in the computing results [7,30]. Liu et al. [7] analyzed the impact of the IRdrop on both training and recall processes: In the training process, an inaccurate memristance can be programmed into a memristor, due to the distorted programming voltage across the device; In the recall process, the effective bitline current relies on not only the summation of the weighted input signals but also the physical distance of these input signals to the peripheral circuits (e.g., WL driver and BL output circuit), which is closely related to the operation condition. To mitigate the impact of such an unpredictable situation, Liu et al. proposed to include an extra row of memristors into the crossbar array. The function of these cells is to adaptively and actively compensate the voltage degradation along the wires and mitigate the impact of the IR-drop. The work effectively enhances the robustness of memristor-based NCS.

3.3.2 System-level solutions for drifting. Various approaches were proposed to recover the memristance level due to drifting. The principle of these methods indeed is refreshing.

Refreshing periodically. The online refreshing is a straightforward strategy to overcome the memristance drifting. Like DRAM designs in main memory, we may adjust the memristances (weights) of a memristor-based NCS back to their initial states periodically. The refreshing can be carried out through programing or online training from scratch. Liu et al. [31], on the other hand, applied a subset of selective training vectors on the memristor arrays in order to recover the resistances precisely and quickly. As the method does not monitor the memoristor states, a conservative refresh period was selected which results in relatively high overheads in refresh time and power consumption. Similarly, Prezioso et al. [32] proposed a on-chip modified training method, which directly applies the voltage representing the differential value on the memristor and thereby completed weight update.

Detect and restore. Tosson et al. [24] proposed a framework to detect the dynamic failures as well as to restore the memristor device. The detect stage records the number of the output pulses. When the 


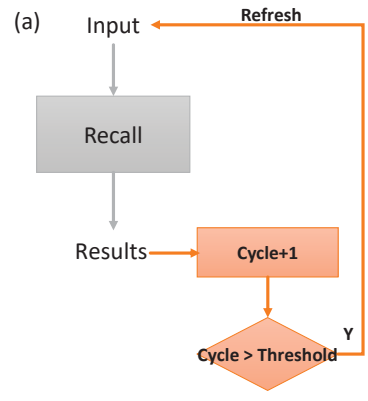

(b)

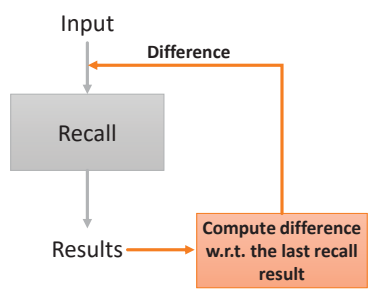

Figure 8: The comparison of refresh techniques (a) at the system level [31] and (b) at the circuit level [10].

amount of the pulse drops to a preset threshold, the dynamic failure is observed and the restore phase will be activated. The restore operation applied a small voltage pulse with the reversed polarity on the memristor device through the output port, to switch the drifted memristor back to its initial state. The framework requires extra detection unit and modifies the write circuit to perform the restore action.

3.3.3 Circuit-level solution for drifting. The refreshing methods at system levels require either an approximation on the refreshing period or a dense monitor/detect on the device access status. As can be seen that these approaches are neither accurate nor easy. Yan et al. [10] presented a close-loop scheme which applied the selfadaptive and tiny compensation currents to refresh the memristor state during the iterative recall operations. Figure 8 illustrates the differences of the refreshing schemes at the architectural and circuit levels.

The work quantitatively investigated the relationship of weights disturbance with the recall conditions. The experiments show that both the resistance value of the memristor and the operation condition will affect the weight disturbance in recall process. For example, a device with the high (or low) resistance is more susceptible to current perturbation when the recall voltage is along the direction of turning "On" (or "Off") the device. Based on this observation, a close-loop design was proposed which can adaptively adjust the sensing conditions to alleviate the impact of memristance drift and improve the system reliability. The key component is a feedback controller which manages the sensing conditions. Specifically, the feedback controller first computes the difference between the actual output bit at column $j$ and then make the decision to adjust the voltage condition to compensate the memristance drift. In the compensating operation, all the weights on column $j$ are decreased if the output bit on column $j$ in the previous recall stage is -1 . Otherwise the output bit on column $j$ in the previous recall stage is 1 , the weights on this column increase.

The principle behind is the "arrogant principle" by the authors, which assumes the predicted result (recall output) is always correct so it can be used as the label in training to determine the sensing condition. Considering the randomness of test samples, the arrogant principle is supported by explanation of expectation. The system starts with a crossbar configuration representing a well-trained network. So the probability of correct predictions is high.

Figure 9 compares the accuracy degradation with respective to the recall number. Conventional open-loop system is not robust

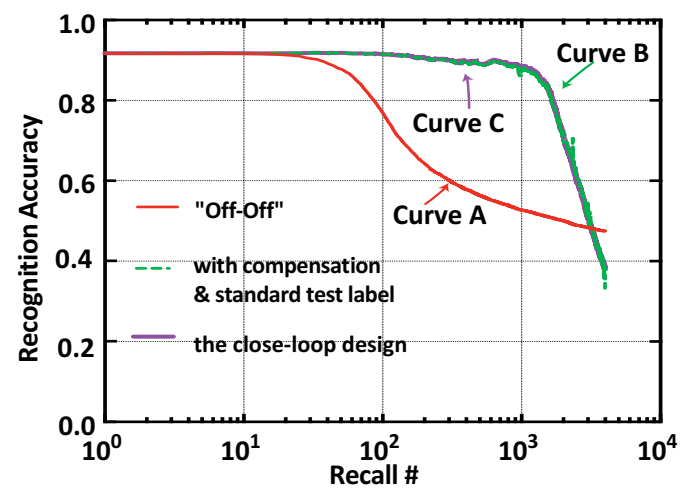

Figure 9: The comparison of the accuracy degradation of the open-loop design, the compensation with test labels and the closed-loop design [10].

enough to combat the memristance drift. Curve A shows the accuracy declines from $91.91 \%$ to $70.01 \%$ after only 137 times of the "Off-Off" recall. Curve B (the green line) presents the compensation scheme using the real labels from the test data set to control the sensing condition. This case indicates the best performance that can be obtained by the ideal memristor-based NCS. Curve $\mathrm{C}$ is the result of the close-loop method where the feedback controller accepts previous recall results to decide the next operation. Comparing Curve B with Curve C, their accuracy difference is tiny (averagely $0.09 \%$ ) by replacing test labels with the previous recall outputs. The closed-loop design dramatically mitigates the degradation of the system accuracy.

The feedback controller is implemented with a winner-take-allcircuit with a few AND gates [33] as it is only in charge of the voltage polarity in recall operations. The extra circuit introduces the $1.61 \%$ additional power consumption and $2.34 \%$ area increase for the single-layer neural network. The recalling method integrates the memristance compensation into the regular recall operation, enhancing the robustness of the memristor device at a small cost of power and area.

\section{DISCUSSION AND CONCLUSION}

The advantages of energy-efficiency, small area and high-density of memristor-based meromorphic computing system make it to be an appealing computation architecture. However, due to the immaturity of memristor technology, complex mechanism of programming and material properties, the static and dynamic reliability issues are common. How to build reliable and efficient neuromorphic design based on memristor technology emerges as the primary challenge.

In this work, we discuss the reliability challenges of the memristorbased NCS and review the solutions at different levels. Here, we separate the reliability issues in memristor-based NCS into categoriesstatic and dynamic. The static issues refer to those that are due to the fabrication flaws or circuit structures. These issues will not change dramatically over time. We notice that the system-level solutions are effective for the static reliability issues. For example, the remapping in $[27,29]$ and the retraining approaches in [8] considered the distribution of weights of neural network models and the distribution of device defects. The dynamic reliability issues, on the other hand, denote the conditions that could be dynamically 
changed according to operation conditions. The system-level approaches [24, 31, 32] help alleviate the impact but cannot be well adapted to the changes in operation conditions. In the situation, the circuit-level solutions, such as the IR-drop compensation through array modification [7] and the recall based on arrogant principle in [10], are more effective.

Considering the development of technology and application, the continually increased data traffic and computation scale put forward more stringent requirements on the reliability and efficiency of the memristor-based NCS. The connection of the memristor device fabrication, memristor circuit design, as well as computation algorithm will be the essential trend. We envision that reliable and efficient memristor-based NCS designs can help promote the use to more applications and industries.

\section{ACKNOWLEDGMENTS}

This work was supported in part by NSF XPS-1744077 and AFRL FA8750-15-2-0048. Bing Li acknowledges the National Academy of Sciences (NAS), USA for awarding the NRC research fellowship. Any opinions, findings and conclusions or recommendations expressed in this material are those of the authors and do not necessarily reflect the views of NSF, AFRL or their contractors.

\section{REFERENCES}

[1] Bing Chen, Fuxi Cai, Jiantao Zhou, Wen Ma, Patrick Sheridan, and Wei D Lu. Efficient in-memory computing architecture based on crossbar arrays. In 2015 IEEE International Electron Devices Meeting (IEDM), pages 17-5, 2015.

[2] Miao Hu, John Paul Strachan, Zhiyong Li, Emmanuelle M Grafals, Noraica Davila, Catherine Graves, Sity Lam, Ning Ge, Jianhua Joshua Yang, and R Stanley Williams. Dot-product engine for neuromorphic computing: programming $1 \mathrm{t} 1 \mathrm{~m}$ crossbar to accelerate matrix-vector multiplication. In 2016 53nd ACM/EDAC/IEEE Design Automation Conference (DAC), pages 1-6, 2016.

[3] B. Li, L. Song, F. Chen, X. Qian, Y. Chen, and H. H. Li. Reram-based accelerator for deep learning. In 2018 Design, Automation Test in Europe Conference Exhibition (DATE), pages 815-820, March 2018.

[4] Dimin Niu, Cong Xu, Naveen Muralimanohar, Norman P Jouppi, and Yuan Xie. Design of cross-point metal-oxide reram emphasizing reliability and cost. In 2013 IEEE/ACM International Conference on Computer-Aided Design (ICCAD), pages 17-23, 2013.

[5] Annie Foong and Frank Hady. Storage as fast as rest of the system. In 2016 IEEE 8th International Memory Workshop (IMW), pages 1-4, 2016.

[6] Chenchen Liu, Bonan Yan, Chaofei Yang, Linghao Song, Zheng Li, Beiye Liu, Yiran Chen, Hai Li, Qing Wu, and Hao Jiang. A spiking neuromorphic design with resistive crossbar. In 2015 52nd ACM/EDAC/IEEE Design Automation Conference (DAC), pages 1-6, 2015.

[7] Beiye Liu, Hai Li, Yiran Chen, Xin Li, Tingwen Huang, Qing Wu, and Mark Barnell. Reduction and ir-drop compensations techniques for reliable neuromorphic computing systems. In 2014 IEEE/ACM International Conference on ComputerAided Design (ICCAD), pages 63-70, 2014.

[8] Chenchen Liu, Miao Hu, John Paul Strachan, and Hai Li. Rescuing memristorbased neuromorphic design with high defects. In Design Automation Conference (DAC), 2017 54th ACM/EDAC/IEEE, pages 1-6. IEEE, 2017.

[9] Ting Chang, Sung-Hyun Jo, and Wei Lu. Short-term memory to long-term memory transition in a nanoscale memristor. ACS Nano, 5(9):7669-7676, 2011.

[10] Bonan Yan, Jianhua (Joshua) Yang, Qing Wu, Yiran Chen, and Hai (Helen) Li. A closed-loop design to enhance weight stability of memristor based neural network chips. In Proceedings of the 36th International Conference on ComputerAided Design, ICCAD '17, pages 541-548, Piscataway, NJ, USA, 2017. IEEE Press.

[11] Yiran Chen, Hai Li, Xiaobin Wang, Wenzhong Zhu, Wei Xu, and Tong Zhang. A nondestructive self-reference scheme for spin-transfer torque random access memory (stt-ram). In 2010 Design, Automation \& Test in Europe Conference \& Exhibition (DATE), pages 148-153, 2010.

[12] Dimin Niu, Yang Xiao, and Yuan Xie. Low power memristor-based reram design with error correcting code. In 2012 17th Asia and South Pacific Design Automation Conference (ASP-DAC), pages 79-84, 2012.

[13] Manqing Mao, Pai-Yu Chen, Shimeng Yu, and Chaitali Chakrabarti. A multilayer approach to designing energy-efficient and reliable reram cross-point array system. IEEE Transactions on Very Large Scale Integration (VLSI) Systems,
25(5):1611-1621, 2017

[14] Amirali Ghofrani, Miguel Angel Lastras-Montaño, and Kwang-Ting Cheng. Towards data reliable crossbar-based memristive memories. In Test Conference (ITC), 2013 IEEE International, pages 1-10. IEEE, 2013.

[15] Leon Chua. Memristor-the missing circuit element. IEEE Transactions on Circuit Theory, 18(5):507-519, 1971

[16] Dmitri B Strukov, Gregory S Snider, Duncan R Stewart, and R Stanley Williams. The missing memristor found. Nature, 453(7191):80-83, 2008.

[17] Cong Xu, Dimin Niu, Naveen Muralimanohar, Rajeev Balasubramonian, Tao Zhang, Shimeng Yu, and Yuan Xie. Overcoming the challenges of crossbar resistive memory architectures. In 2015 IEEE 21st International Symposium on High Performance Computer Architecture (HPCA), pages 476-488. IEEE, 2015.

[18] Cong Xu, Xiangyu Dong, Norman P Jouppi, and Yuan Xie. Design implications of memristor-based rram cross-point structures. In Design, Automation \& Test in Europe Conference \& Exhibition (DATE), 2011, pages 1-6. IEEE, 2011.

[19] Miao Hu, Hai Li, Yiran Chen, Qing Wu, and Garrett S Rose. Bsb training scheme implementation on memristor-based circuit. In Computational Intelligence for Security and Defense Applications (CISDA), 2013 IEEE Symposium on, pages 80-87. IEEE, 2013.

[20] Pai-Yu Chen and Shimeng Yu. Compact modeling of rram devices and its applications in $1 \mathrm{t} 1 \mathrm{r}$ and $1 \mathrm{~s} 1 \mathrm{r}$ array design. IEEE Transactions on Electron Devices, 62(12):4022-4028, 2015.

[21] John Paul Strachan, Antonio C Torrezan, Feng Miao, Matthew D Pickett, J Joshua Yang, Wei Yi, Gilberto Medeiros-Ribeiro, and R Stanley Williams. State dynamics and modeling of tantalum oxide memristors. IEEE Transactions on Electron Devices, 60(7):2194-2202, 2013.

[22] S. Li, W. Wen, Y. Wang, S. Han, Y. Chen, and H. Li. An fpga design framework for cnn sparsification and acceleration. In 2017 IEEE 25th Annual International Symposium on Field-Programmable Custom Computing Machines (FCCM), pages 28-28, April 2017.

[23] Indranil Chakraborty, Deboleena Roy, and Kaushik Roy. Technology aware training in memristive neuromorphic systems for nonideal synaptic crossbars. IEEE Transactions on Emerging Topics in Computational Intelligence, 2(5):335-344, 2018.

[24] Amr Tosson, Shimeng Yu, Mohab Anis, and Lan Wei. Mitigating the effect of reliability soft-errors of rram devices on the performance of rram-based neuromorphic systems. In Proceedings of the on Great Lakes Symposium on VLSI 2017, pages 53-58. ACM, 2017.

[25] Chaofei Yang, Beiye Liu, Hai Li, Yiran Chen, Wujie Wen, Mark Barnell, Qing Wu, and Jeyavijayan Rajendran. Security of neuromorphic computing: thwarting learning attacks using memristor's obsolescence effect. In Proceedings of the 35th International Conference on Computer-Aided Design, page 97, 2016.

[26] An Chen and Ming-Ren Lin. Variability of resistive switching memories and its impact on crossbar array performance. In Reliability Physics Symposium (IRPS), 2011 IEEE International, pages MY-7. IEEE, 2011.

[27] Wenqin Huangfu, Lixue Xia, Ming Cheng, Xiling Yin, Tianqi Tang, Boxun Li, Krishnendu Chakrabarty, Yuan Xie, Yu Wang, and Huazhong Yang. Computationoriented fault-tolerance schemes for rram computing systems. In ASP-DAC, pages 794-799, 2017.

[28] Lixue Xia, Mengyun Liu, Xuefei Ning, Krishnendu Chakrabarty, and Yu Wang. Fault-tolerant training with on-line fault detection for rram-based neural computing systems. In Proceedings of the 54th Annual Design Automation Conference 2017, page 33. ACM, 2017.

[29] L. Xia, W. Huangfu, T. Tang, X. Yin, K. Chakrabarty, Y. Xie, Y. Wang, and H. Yang. Stuck-at fault tolerance in rram computing systems. IEEE fournal on Emerging and Selected Topics in Circuits and Systems, 8(1):102-115, March 2018.

[30] Pai-Yu Chen, Deepak Kadetotad, Zihan Xu, Abinash Mohanty, Binbin Lin, Jieping Ye, Sarma Vrudhula, Jae-sun Seo, Yu Cao, and Shimeng Yu. Technology-design co-optimization of resistive cross-point array for accelerating learning algorithms on chip. In Proceedings of the 2015 Design, Automation \& Test in Europe Conference \& Exhibition, pages 854-859. EDA Consortium, 2015.

[31] X. Liu, M. Mao, B. Liu, B. Li, Y. Wang, H. Jiang, M. Barnell, Q. Wu, J. Yang, H. Li, and Y. Chen. Harmonica: A framework of heterogeneous computing systems with memristor-based neuromorphic computing accelerators. IEEE Transactions on Circuits and Systems I: Regular Papers, 63(5):617-628, 2016.

[32] Mirko Prezioso, Farnood Merrikh-Bayat, BD Hoskins, GC Adam, Konstantin K Likharev, and Dmitri B Strukov. Training and operation of an integrated neuromorphic network based on metal-oxide memristors. Nature, 521(7550):61, 2015.

[33] Joongho Choi and Bing J Sheu. A high-precision vlsi winner-take-all circuit for self-organizing neural networks. IEEE fournal of Solid-state circuits, 28(5):576-584, 1993. 